\title{
Armed and attentive: Holding a weapon can bias attentional priorities in scene viewing
}

\author{
Adam T. Biggs • James R. Brockmole • Jessica K. Witt
}

Published online: 12 September 2013

(C) Psychonomic Society, Inc. 2013

\begin{abstract}
The action-specific perception hypothesis (Witt, Current Directions in Psychological Science 20: 201-206, 2011) claims that the environment is represented with respect to potential interactions for objects present within said environment. This investigation sought to extend the hypothesis beyond perceptual mechanisms and assess whether actionspecific potential could alter attentional allocation. To do so, we examined a well-replicated attention bias in the weapon focus effect (Loftus, Loftus, \& Messo, Law and Human Behaviour 1, 55-62, 1987), which represents the tendency for observers to attend more to weapons than to neutral objects. Our key manipulation altered the anticipated actionspecific potential of observers by providing them a firearm while they freely viewed scenes with and without weapons present. We replicated the original weapon focus effect using modern eye tracking and confirmed that the increase in time looking at weapons comes at a cost of less time spent looking at faces. Additionally, observers who held firearms while viewing the various scenes showed a general bias to look at faces over objects, but only if the firearm was in a readily usable position (i.e., pointed at the scenes rather than holstered at one's side). These two effects, weapon focus and the newly found bias to look more at faces when armed, canceled out one another without interacting. This evidence confirms that the action capabilities of the observer alter more than just perceptual mechanisms and that holding a weapon can change attentional priorities. Theoretical and real-world implications are discussed.
\end{abstract}

\footnotetext{
A. T. Biggs ( $\square)$

Department of Psychology \& Neuroscience, Center for Cognitive Neuroscience, Duke University, B203 LSRC, Box 90999 Durham, NC 27708-0754, USA

e-mail: adam.biggs@duke.edu

J. R. Brockmole

University of Notre Dame, Notre Dame, USA

J. K. Witt

Colorado State University, Fort Collins, USA
}

Keywords Attention - Cognitive and attentional control . Eye movements and visual attention

The allocation of overt attention within a scene is determined by a variety of interacting factors, including episodic memory for that scene's structure and contents (e.g., Brockmole \& Henderson, 2006a, b; Castelhano \& Henderson, 2007; Eckstein, Drescher, \& Shimozaki, 2006), schematic knowledge of the typical structure and content found within similar scenes (e.g., Antes, 1974; Henderson, Weeks, \& Hollingworth, 1999; Loftus \& Mackworth, 1978), and taskrelated knowledge pertaining to one's goals when viewing the scene (e.g., Castelhano, Mack, \& Henderson, 2009; Hayhoe, Shrivastava, Mruczek, \& Pelz, 2003; Yarbus, 1967). In this report, we consider an underexplored factor that may also modify the allocation of attention in a scene: the actionrelated capabilities of the observer.

Our hypothesis - that one's ability to engage in certain kinds of actions may influence attention - is derived from recent work linking action and perception. To take one example, the theory of event coding (Hommel, Müsseler, Aschersleben, \& Prinz, 2001) posits that both perceptual and action-based representations arise from a common code. On this view, perception and action planning involve shared processes that can facilitate or inhibit each other. For example, planning or executing directional hand movements influences the perceived directional motion and orientation of an object (Müsseler \& Hommel, 1997; Zwickel, Grosjean, \& Prinz, 2007). A similar effect describes how the ability to identify tools is disrupted if the motor processes required to act on the tools are otherwise engaged (Witt, Kemmerer, Linkenauger, \& Culham, 2010). Hence, if an object is being used by an individual, it will be incorporated into the action representation and will have consequences for perceptual processing (Iriki, Tanaka, \& Iwamura, 1996; Miles \& Proctor, 2011).

A related perspective, known as the action-specific perception hypothesis (Witt, 2011), claims that the perceptual system represents the environment in terms of one's ability to interact 
with the objects present. As such, perception is influenced by an individual's physical attributes, current capabilities, and recent performance. Evidence supporting the action-specific perception hypothesis comes from numerous empirical sources, including studies showing that people with wider shoulders perceive doorways to be narrower (Stefannuci \& Geuss, 2009), people batting better than others perceive softballs to be larger (Gray, 2012; Witt \& Proffitt, 2005), and people using reachextending tools perceive objects to be closer than when a tool is not used (Davoli, Brockmole, \& Witt, 2012; Osiurak, Morgado, \& Palluel-Germain, 2012; Witt, Proffitt, \& Epstein, 2005). Beyond the perception of spatiotemporal properties, object recognition can also be distorted by potential interactions with the environment. For example, holding a gun leads to a bias for observers to perceive guns in the hands of others (Witt \& Brockmole, 2012).

Here, we ask whether the effects of action are limited to perceptual mechanisms or whether they also apply to attentional control. Does an observer's representation of his or her ability to act on objects in the world alter the manner in which he or she allocates attention to various objects in a scene? We addressed this question by looking at attentional engagement and allocation in the context of viewing scenes where someone held either a weapon (e.g., a gun) or a neutral object (e.g., a remote control). The presence of weapons in a scene leads to a well-known cognitive bias called the weapon focus effect. While predominantly described as a memory effect where the presence of weapons in a scene leads to a reduced ability to recall details about the perpetrator (see Fawcett, Russell, Peace, \& Christie, 2013, for a review), it is also apparent at an attentional level, since weapons in a scene receive more fixations and longer fixations than do other hand-held objects, such as personal checks (Loftus, Loftus, \& Messo, 1987). Our goal was to determine whether this attentional bias could be modulated by giving observers a weapon of their own.

By arming an individual with a gun, his or her repertoire of possible actions is significantly changed; hence, new attentional priorities may be set during scene viewing. How might arming an individual affect attentional allocation in general and the weapon focus effect in particular? There are several possibilities to consider. The first, of course, is that holding a gun may have no effect on attention. Whereas the perceptual system is influenced by action, attentional systems may not be sensitive to the behavioral repertoire of the perceiver. This result would indicate that the previous conclusions of the action-specific perception hypothesis cannot be theoretically extended to attentional control.

If an action-attention interface exists, the magnitude of the weapon focus effect may be altered in one of two ways. One possibility is that an armed individual may exhibit a bias to treat all objects as more weapon-like (cf. Witt \& Brockmole, 2012). As such, attention to neutral objects may increase toward the levels normally observed with weapons, indicative that the observer is biased to attend to all objects as though they were weapons. On the other hand, certain gun rights advocates suggest that being armed can lead to a feeling of protection despite facing a dangerous situation (Miller, 2013). ${ }^{1}$ If supported, wielding a gun could have the effect of decreasing the attention typically allotted toward weapons to levels seen with neutral objects. In other words, weapons would be treated more like neutral objects because they would seem less threatening.

A fourth possibility is that individuals view the world in terms of affordances, or possibilities for action (Gibson, 1979), and armed individuals view the world in terms of the affordances that result from wielding a gun, just as unarmed individuals view the world in terms of affordances available at that time. When unarmed and viewing a person wielding a gun, an individual must decide how to act in order to avoid injury from the weapon held by another individual. This caveat makes any weapon a substantially more important aspect of a scene. When armed, however, an individual must decide whether to utilize their expanded behavioral repertoire that results from wielding a gun. This caveat makes other aspects of a scene, such as facial expressions, potentially more informative because they may reveal the other person's intent. In this case, a shift may be observed where attention is moved from a perpetrator's weapon to his or her face.

These possible outcomes are investigated in Experiments 1 and 2. Any outcome in which arming an individual modulates his or his attentional allocation would be consistent with an action-specific influence on attentional control. Moreover, the outcome will determine how action influences attention, as well as suggest the underlying mechanism, for which we outlined three possibilities: (1) a bias for neutral objects to be attended to as if they were weapons, (2) a bias for weapons to be attended to as if they were neutral objects, (3) a bias to detect the most relevant affordances in the environment, with relevancy being determined in part by one's own abilities.

\section{Experiment 1}

Experiment 1 had two goals. The first was to replicate previous demonstrations of weapon focus using eye-tracking methodologies. Twenty-six years ago, Loftus et al. (1987) used video-based techniques to show that observers spend more time fixating weapons than neutral objects in a scene. This is an important result that explicitly links weapon focus to shifts in overt attention allocation. We therefore wanted to replicate

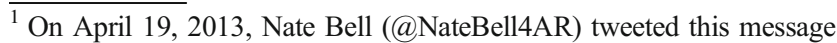
following the bombing of the Boston Marathon: "I wonder how many Boston liberals spent the night cowering in their homes wishing they had an AR-15 with a hi-capacity magazine?" The tweet was in response to the manhunt for the Boston Marathon bombers and has since been removed from Twitter.
} 
this finding using modern eye-tracking technologies. We further wanted to replicate previous findings that suggest that the bias to attend to weapons results in decreased attentional allocation to other objects in the same scene. When studied as a memory effect, the presence of a weapon impairs the ability to correctly describe a perpetrator's physical features, as well as the subsequent ability to identify him or her (Tooley, Brigham, Maass, \& Bothwell, 1987). To root this deficit in terms of attentional control, we analyzed not only fixations devoted to the weapon, but also those directed to the actor's face. Not only should weapons be fixated longer than neutral objects across scenes, their presence should be accompanied by a decrease in attention to the corresponding faces.

\section{Method}

\section{Participants}

Eighteen undergraduate students from the University of Notre Dame participated in exchange for course credit. All observers reported normal or corrected-to-normal visual acuity.

\section{Stimuli and apparatus}

Stimuli consisted of 38 full-color digital photographs that each depicted one person. The use of digital photography to study weapon focus both has precedent in the literature (e.g., Hope \& Wright, 2007; Loftus et al., 1987) and facilitates the analysis of eye movements relative to video-based methods. In half of the images, the actor held a single neutral (i.e., not a weapon) object such as a remote control, bouquet of flowers, or drink. In the other half of the images, the actor held a single weapon such as a gun or a knife. Each image was presented on a 21-in. CRT display with a refresh rate of $120 \mathrm{~Hz}$ and at a resolution of $800 \times 600$ pixels. Images were chosen to depict a variety of facial emotions and environmental contexts both for scenes containing a weapon and for those containing a neutral object. People displayed in each image were neither famous nor associated with the project, so as to avoid any familiarity between participants and people displayed in the images.

Stimuli were viewed at a constrained distance of $81 \mathrm{~cm}$. Observer's eye movements were sampled at a rate of $1000 \mathrm{~Hz}$ with an EyeLink 2K eye-tracking system (SR Research, Inc.) running in pupil and corneal reflection mode, resulting in an average spatial accuracy of $0.15^{\circ}$. During the experiment, observers held a metallic cylinder $15 \mathrm{~cm}$ in length and $1.4 \mathrm{~cm}$ in diameter. Participants rested their hands on the table, while holding the metal cylinder in their dominant hand for the duration of the experiment. This placed a control object in the observer's hand while viewing the images and permitted a more direct comparison with Experiment 2, where the cylinder was replaced with a gun. Hence, in each experiment, observers held a similarly weighted object throughout the testing session to rule out physical strain or fatigue as potential causes of behavioral differences across conditions. However, the metal cylinder is too small to be used as a weapon with any practical effectiveness. ${ }^{2}$

\section{Design and procedure}

Throughout the experiment, participants held the cylinder in their dominant hand while seated at the eyetracker. Each participant viewed the 19 images in which actors held neutral objects and the 19 images in which actors held weapons. These image types were intermixed during the trial sequence and were presented to each participant in a different random order. Each image was freely viewed for $5 \mathrm{~s}$, whereupon it was removed and replaced with a central fixation cross. A buttonpress caused the next image to appear on-screen. Hence, the progression through the trials was self-paced. Participants were informed ahead of time that some of the images would contain weapons and that some of the images would not.

\section{Results}

Within each image, two regions of interest were defined. The face region and the object region were defined by the smallest imaginary bounding boxes that completely circumscribed the actor's face and the object in his or her hand, respectively. There was no spatial overlap between the face and object regions in any image. The average size of neutral objects and weapons was equated, as was the size of faces across images containing neutral objects and those containing weapons. Fixations on each image were sorted according to whether they fell within one or the other of these critical regions. The dependent variables were the dwell time (i.e., the sum of all fixation durations) and fixation count (i.e., number of fixations) within each region. Although not independent, these analyses collectively reveal the relative weight observers placed on the actor's face and on the object in the actor's hand while allocating attention to the display (see Fig. 1).

\section{Dwell times}

A 2 (critical region: face or object) $\times 2$ (viewed object: weapon or neutral) repeated measures analysis of variance (ANOVA) showed that observers looked at the actor's face longer $(M=2,008 \mathrm{~ms})$ than they looked at the object in the actor's hand $(M=1,157 \mathrm{~ms}), F(1,17)=73.41, p<.001, \eta_{\mathrm{p}}{ }^{2}=.81$. Importantly, however, the amount of time spent looking at faces and objects depended on scene content, $F(1,17)=14.69$, $p<.001, \eta_{\mathrm{p}}^{2}=.46$. Specifically, weapons were looked at longer

\footnotetext{
${ }^{2}$ We verified this assertion empirically by asking a group of 8 individuals to indicate how useful the cylinder would be as a weapon on a scale of 1 (low) to 7 (high). The average response was 2.75; this rating was similar to that of staplers and coffee mugs. When asked about guns, the average response was, not surprisingly, 7.
} 


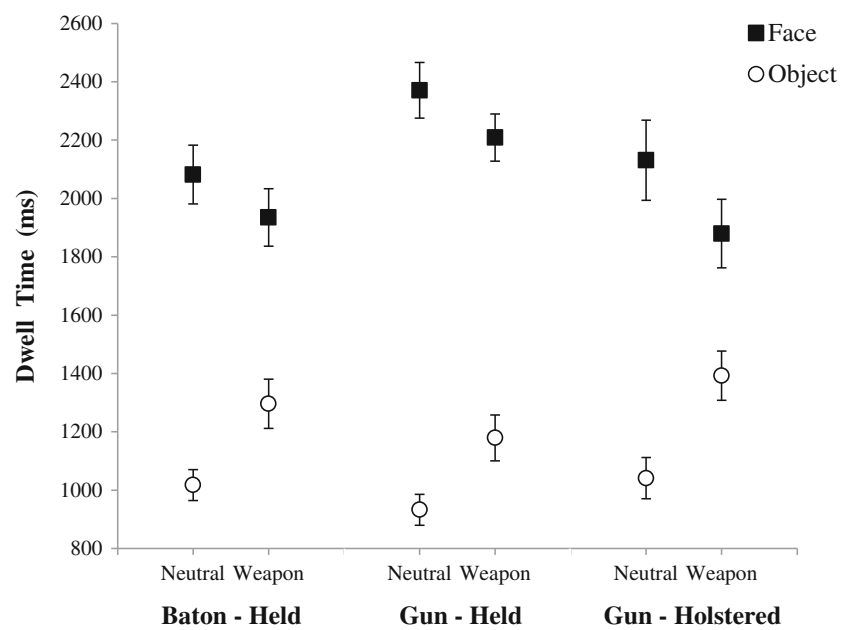

Fig. 1 Dwell times as a function of object held by the participant (BatonHeld, Experiment 1; Gun-Held, Experiment 2; Gun-Holstered, Experiment 3), region of interest (object or face), and object held by the actor in the viewed scene (neutral or weapon). The weapon focus effect is the shift to looking longer at the object when it is a weapon, which results in less time spent looking at the face. This bias is still present when the participant holds a gun, yet there is also a shift to looking longer at faces. Thus, the distribution of dwell times is similar for holding a control object and looking at a neutral object as for holding a gun and looking at a gun. Error bars are 1 SEM calculated within subjects for each experiment

$(M=1,296 \mathrm{~ms})$ than neutral objects $(M=1,018 \mathrm{~ms}), t(17)=$ $4.41, p<.001$, and this increase in looking to weapons came at the cost of looks to the face. When the actor held a weapon, observers spent less time looking at the face region $(M=1,935$ $\mathrm{ms}$ ) than they did when the actor held a neutral object $(M=2,082 \mathrm{~ms}), t(17)=2.74, p=.01$.

\section{Fixation count}

Parallel analyses were conducted on fixation counts. Observers made more fixations to the actor's face $(M=6.01$ fixations) than they made to the object in the actor's hand $\left(M=3.87\right.$ fixations), $F(1,17)=87.90, p<.001, \eta_{\mathrm{p}}{ }^{2}=.84$. Importantly, the number of fixations made to faces and objects depended on scene content, $F(1,17)=15.71$, $p<.001, \eta_{\mathrm{p}}{ }^{2}=.48$. Specifically, weapons were looked at more $(M=4.40$ fixations) than neutral objects $(M=3.33$ fixations), $t(17)=5.97, p<.001$. In contrast, when a weapon was present, observers made fewer fixations on the face region ( $M=5.88$ fixations) than they did when the actor held a neutral object ( $M=6.14$ fixations), although the difference was nonsignificant, $t(17)=1.29, p=.22$.

\section{Discussion}

This trade-off between attentional allocation to weapons and other aspects of a scene, especially important aspects such as faces, is consistent with the weapon focus effect. The classic finding was observed through both dwell times and fixation count, which successfully replicates the weapon focus effect using modern eye-tracking technology. In Experiment 2, we examined whether the magnitude of this bias is altered when observers are armed.

\section{Experiment 2}

The purpose of Experiment 2 was to explicitly test the hypothesis that attention allocation in a scene is determined in part by the action-specific potential of the observer. In particular, weapons change the way in which an observer can act in relation to other people. We determined whethere this change in action-specific potential would alter attentional allocation, relative to Experiment 1. To this end, Experiment 2 replicated the procedures used in Experiment 1, except that, in this case, all participants held a firearm while viewing the scenes. Participants were instructed to hold the firearm pointed toward the display screen while viewing the scenes. This posture mimicked a typical stance one would assume if using a firearm in a real-world scenario. That is, if participants are going to be viewing scenes where another individual could have a weapon, they would presumably be in better position to act upon a changed action-specific potential due to the weapon (e.g., presumably, to defend themselves), when the weapon is readily usable. Although the firearm used in this experiment was nonfunctional and was not used to make any kind of overt response, we argue that the act of holding a weapon in a manner consistent with its use may nevertheless give rise to an altered representation of one's action-related capabilities.

Method

\section{Participants}

A new sample of 18 undergraduate students from the University of Notre Dame participated in exchange for course credit. All observers reported normal or corrected-to-normal visual acuity.

\section{Stimuli, apparatus, and procedure}

All aspects of the stimuli, apparatus, and procedure were the same as in Experiment 1, except that during the experiment, observers held a .177-caliber $\mathrm{CO}_{2}$-powered $\mathrm{BB}$ pistol, which was rendered nonfunctional by the Notre Dame Security Police Department. Participants were informed that the firearm had been rendered nonfunctional, but not what type of ammunition it fired. The participants were instructed to hold the firearm in their dominant hand and to point it at the display. Similar to Experiment 1, their hands rested on a table, but all participants were instructed to keep the firearm upright and pointed at the screen for the duration of the experiment. 
Although the firearm was pointed at the screen, it did not block their field of view.

\section{Results}

A 2 (critical region: face or object) $\times 2$ (viewed object: weapon or neutral) repeated measures ANOVA was conducted for dwell times (how long the observer spent looking at a given region of interest) and fixation count (number of fixations made within a given region of interest).

\section{Dwell times}

Initial analyses mirrored those conducted in Experiment 1 and revealed qualitatively similar results (see Fig. 1). Observers looked at the actor's face longer $(M=2,290 \mathrm{~ms})$ than they looked at the object in the actor's hand $(M=1,056$ $\mathrm{ms}), F(1,17)=133.24, p<.001, \eta_{\mathrm{p}}{ }^{2}=.89$, and the amount of time spent looking at either region depended on the nature of the viewed object, $F(1,17)=12.33, p<.01, \eta_{\mathrm{p}}{ }^{2}=0.42$. The presence of a weapon in the actor's hand led to an increased amount of looking to the object (weapons, $M=1,179 \mathrm{~ms}$; neutral objects, $M=933 \mathrm{~ms}), t(17)=3.79, p<.001$, and a decreased amount of looking to the face (weapon present, $M=2,209 \mathrm{~ms}$; neutral object present, $M=2,371 \mathrm{~ms}$ ), $t(17)=2.68, p=.02$. Once again, when the actor held a weapon in the scene, the participants' bias was to look at weapons for longer and faces for shorter periods of time, which is consistent with weapon focus.

\section{Fixation count}

Observers made more fixations to the actor's face $(M=6.26$ fixations) than they made to the object in the actor's hand $\left(M=3.42\right.$ fixations), $F(1,17)=97.61, p<.001, \eta_{\mathrm{p}}{ }^{2}=.85$. Importantly, the number of fixations made to faces and objects depended on scene content, $F(1,17)=22.08, p<.001$, $\eta_{\mathrm{p}}{ }^{2}=.57$. Specifically, weapons were looked at more ( $M=3.80$ fixations) than neutral objects ( $M=3.03$ fixations), $t(17)=4.40, p<.001$. When the actor held a weapon, observers made fewer fixations on the face region $(M=5.98$ fixations) than they did when the actor held a neutral object ( $M=6.54$ fixations), $t(17)=3.25, p<.01$.

\section{Effect of action}

Whether holding a gun (Experiment 2) or a neutral cylinder (Experiment 1), observers were biased to allocate attention to weapons present in a display at the cost of attending to faces. To contrast potential quantitative differences in looking behavior between the groups, we conducted a 2 (critical region: face or object) $\times 2$ (viewed object: weapon or neutral) $\times 2$ (group: observer holding a weapon or a neutral object ${ }^{3}$ ) mixed model ANOVA. The only reliable effect involving the group factor was an interaction with critical region for dwell times, $F(1,34)=6.85, p<$ $.05, \eta_{\mathrm{p}}^{2}=.17$, all other $p \mathrm{~s}>.45$. When handling a gun themselves, observers spent $101 \mathrm{~ms}$ less time looking at the object region than they did when they held the neutral cylinder, although the difference was nonsignificant, $t(34)=1.17, p=$ .25. Conversely, when handling a gun, observers spent $282 \mathrm{~ms}$ more time looking at the face region than they did when holding the cylinder, $t(34)=2.21, p<.05$. Thus, observers who are themselves armed shifted their overall distribution of attention by spending less time looking at what another person was holding and more time looking at that person's face.

\section{Discussion}

Armed observers spent less time looking at objects and more time looking at faces than did their unarmed counterparts. Holding a weapon therefore created a bias to look at faces, much as the presence of a weapon created a bias to look at the weapon. The two biases - a weapon focus bias to look at weapons over neutral objects and an armed observer bias to look at faceswere quantitatively similar but operated in opposite directions, which resulted in equivalent time spent looking at faces for the baseline group (observers holding a control object looking at a neutral scene) and for participants holding weapons who viewed a scene where the actor also held a weapon $(p>.5)$.

Although holding a firearm clearly altered participants' attentional allocation, alternative explanations can be postulated that do not rely on representations of actions. Specifically, arming an observer may reduce the unusualness of weapons in a scene. People tend to look more at objects when they are unexpected in a scene (Loftus \& Mackworth, 1978), and because weapons are not generally expected in everyday situations, their unexpected presence could account for a weapon focus effect (Pickel, 1998). To address this issue, we compared the dwell times in the first half of the experiment with dwell times in the second half of the experiment. By the second half of the experiment, participants would have seen numerous images containing weapons after half the experiment is complete. We focused this analysis on dwell times for faces, since the observed change in attentional allocation for armed observers is primarily a shift in attending to faces.

The $2 \times 2$ interaction of experimental session (first half or second half) and weapon presence in the viewed scene (weapon absent versus weapon present) was significant, $F(1,34)=$ $15.63, p<.001, \eta_{\mathrm{p}}{ }^{2}=.32$ (see Fig. 2). In the first half of the experiment, participants attended more to faces when a neutral object was present $(M=2,347 \mathrm{~ms})$ than to faces when a weapon

\footnotetext{
${ }^{3}$ Participants were recruited in parallel for Experiments 1 and 2, with random assignment to each experiment, thereby reducing the likelihood that there are systematic differences between the two subject populations.
} 


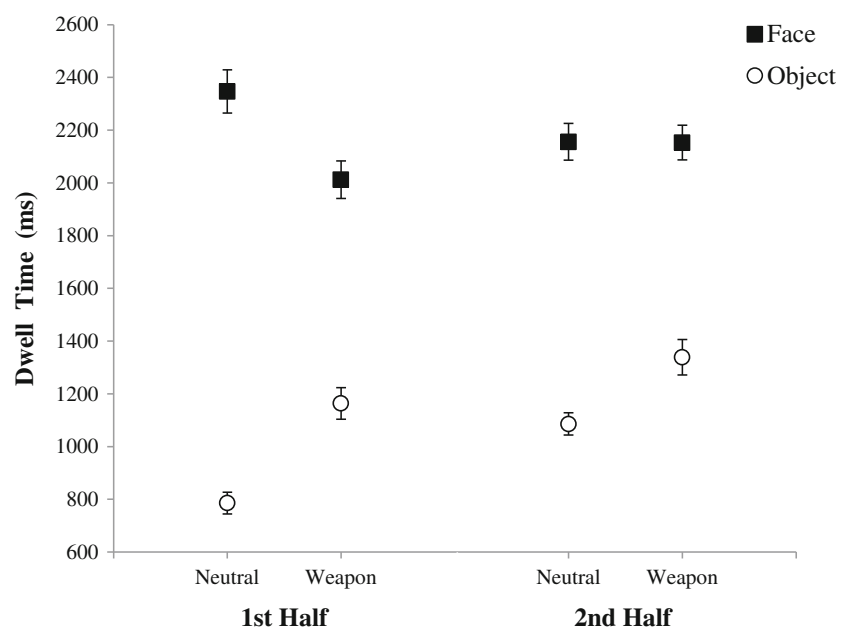

Fig. 2 Dwell times as a function of time (first half of the experimental session or second half of the experimental session), region of interest (object or face) and object held by the actor in the viewed scene (neutral or weapon) for Experiments 1 and 2. The weapon focus effect is the shift to looking longer at the object when it is a weapon, which results in less time spent looking at the face. This bias is still present in both halves of the experiment as far as the object is concerned, but there is no difference in time spent looking at the face during the second half of the experiment

was present $(M=2,012 \mathrm{~ms})$, but there was no difference in the second half of the experiment (time dwelling on faces when a neutral object was present, $M=2,156 \mathrm{~ms}$; time dwelling on faces when a weapon was present, $M=2,153 \mathrm{~ms}$ ). This significant interaction provides support for the hypothesis that unusualness contributes to the weapon focus effect. Most tests of this hypothesis involve comparing a weapon with an unusual item (such as a stalk of celery) and measuring the subsequent effects on memory. Here, we compared weapons with weapons and manipulated unusualness by looking at exposure (initial vs. repeated). We also assessed differences between unusual and usual items at the time of viewing the scene, rather than later effects on memory (see Fawcett et al., 2013). While this provides a new type of evidence for the unusualness hypothesis of the weapon focus effect, our primary interest is in the effects of holding a gun on attention allocation.

The interaction of experimental session and weapon presence did not further interact with whether or not the observer was armed, $F(1,34)=0.07, p=.80$. That is, observers in the second half of the experiment attended more to faces when a weapon was present in the scene if they were armed (as in Experiment 2; $M=2,302 \mathrm{~ms}$ ) than if they were unarmed (as in Experiment 1; $M=2,005 \mathrm{~ms}$ ). Once participants completed half the experiment, they should find the presence of weapons in a scene less unusual, but this reduction in unusualness does not appear to interact with the changed attentional allocation due to the observer being armed. In other words, while repeated exposure to guns reduced the weapon focus effect, repeated exposure to guns did not reduce the effect of holding a gun on attention allocation, thereby supporting the idea that holding a gun and seeing a gun produce two separate biases in attention.
There are, however, further alternative explanations that need to be ruled out before a strong conclusion linking action and attention can be made. For example, an armed individual may experience increased physiological arousal that, in turn, increases attention to the source of the arousal (the weapon) and decreases attention to more peripheral stimuli (cf. Peters, 1988). Alternatively, an armed individual may find weapons in the hands of others less surprising, thereby reducing attention to their weapons (cf. Pickel, 1999). Neither of these explanations specifically rests on the actions available to the observer; instead, they rest on environmental circumstances that may modulate arousal or top-down expectations. These possibilities are addressed in Experiment 3,

\section{Experiment 3}

The purpose of Experiment 3 was to determine whether the mere presence of a firearm, divorced from any action associated with its use, can lead to the same changes in attentional allocation that were observed in Experiment 2 (when observers pointed the weapon at the screen). In Experiment 3, observers were once again armed, but the firearm remained holstered at their side during the experiment. As such, the gun was present (i.e., it could affect arousal and environmental expectations) but not immediately incorporated into the observer's action repertoire (i.e., it was less able to evoke an action-specific response). Any effects observed in Experiment 2 should therefore be muted in Experiment 3 if they were action specific.

\section{Method}

\section{Participants}

Twenty undergraduate students from the University of Notre Dame participated in exchange for course credit. All observers reported normal or corrected-to-normal visual acuity.

\section{Stimuli, apparatus, and procedure}

All aspects of the stimuli, apparatus, and procedure were the same as in Experiment 2, except that the pistol remained holstered on each observer's hip (on their dominant side). The experimenter showed the firearm to each participant and then placed the firearm in the holster. Each participant was asked to use the integrated metal clip to affix the holster to his or her pant-waist. Direct interaction with the firearm was therefore held to a relative minimum.

Results

A 2 (critical region: face or object) $\times 2$ (viewed object: weapon or neutral object) repeated measures ANOVA was conducted 
for dwell times (how long the observer spent looking at a given region of interest) and fixation count (number of fixations made within a given region of interest).

\section{Dwell times}

Observers looked at the actor's face longer $(M=2,005 \mathrm{~ms})$ than they looked at the object in the actor's hand $(M=1,217 \mathrm{~ms})$, $F(1,19)=23.40, p<.001, \eta_{\mathrm{p}}{ }^{2}=.55$, and the amount of time spent looking at either region depended on the nature of the viewed object, $F(1,19)=23.12, p<.01, \eta_{\mathrm{p}}{ }^{2}=.55$. The presence of a weapon in the actor's hand led to an increased amount of looking to the object (weapons, $M=1,393 \mathrm{~ms}$; neutral objects, $M=1,041 \mathrm{~ms}), t(19)=5.13, p<.001$, and a decreased amount of looking to the face (weapon present, $M=1,880 \mathrm{~ms}$; neutral object present, $M=2,131 \mathrm{~ms}$ ), $t(19)=3.28, p<.01$. Once again, when the actor held a weapon in the scene, the participants' bias was to look at weapons for longer and faces for shorter periods of time, which is consistent with weapon focus.

\section{Fixation count}

Observers made more fixations to the actor's face $(M=5.55$ fixations) than they made to the object in the actor's hand ( $M=$ 3.93 fixations), $F(1,19)=18.29, p<.001, \eta_{\mathrm{p}}{ }^{2}=.49$. Importantly, the number of fixations made to faces and objects depended on scene content, $F(1,19)=16.63, p<.001, \eta_{\mathrm{p}}{ }^{2}=$ .47. Specifically, weapons were looked at more $(M=4.53$ fixations) than neutral objects ( $M=3.34$ fixations), $t(19)=$ $5.00, p<.001$. When the actor held a weapon, observers made fewer fixations on the face region ( $M=5.40$ fixations) than they did when the actor held a neutral object $(M=5.70$ fixations), although the difference was nonsignificant, $t(19)=$ $1.56, p=.14$.

\section{Discussion}

When participants were armed in Experiment 2, their attentional allocation strategies changed while viewing scenes. However, participants in Experiment 3 did not show the same bias as those in Experiment 2, despite both sets of participants being armed. The critical difference was whether participants held the firearm in a readily usable position, which likely potentiated the action of shooting. Participants who pointed a weapon at the display exhibited a changed attentional allocation strategy, whereas participants with a holstered weapon showed no difference from unarmed participants (e.g., a direct comparison between dwell time analyses for Experiments 1 and 3 showed no significant differences by experiment, all $p$ s > .30; see Fig. 3). This evidence further supports the actionspecific hypothesis, in that the changed attentional allocation strategy depends upon having the weapon in a position to use it, rather than simply being armed.

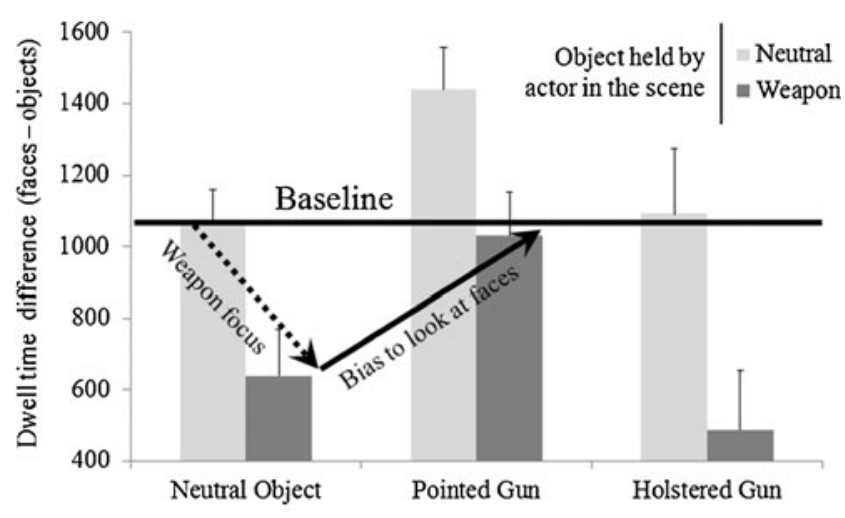

Object held by observer viewing the scene

Fig. 3 Difference in dwell time (in milliseconds) spent looking at faces versus objects as calculated by subtracting dwell time for object region from dwell time for the face region. Error bars are 1 SEM calculated within-subjects for each experiment. Larger differences represent more time spent looking at faces than at objects in the scene. Baseline is indicated by difference in time spent viewing a neutral scene while holding a control object. Weapon focus is indicated by dotted lines, whereas the armed bias to look at faces is indicated by the dashed line

\section{General discussion}

Previous research has shown that perception and action are intimately related (Brockmole, Davoli, Abrams, \& Witt, 2013; Witt, 2011). The present experiments examined whether action-based representations also influence gaze control and, hence, attention allocation. Across three experiments, observers viewed scenes in which an actor held a weapon (e.g., a gun) or a neutral object (e.g., a drink), and we measured the attentional priority given to the actor's face versus the object in his or her hand by recording the observers' eye movements. In Experiment 1, observers were unarmed. In Experiment 2, observers held a replica gun in a defensive posture. In Experiment 3, observers wore a holstered gun on their hip. Observers were biased to attend to weapons present in a scene over neutral objects (i.e., weapon focus) across all three experiments. That said, observers holding a firearm showed an increased bias to attend to faces and a decreased bias to attend to objects of all kinds. Importantly, this object-face trade-off was not apparent when the firearm was holstered, suggesting that the mere possession of a weapon is not sufficient to alter attentional priorities. In summary, under free-viewing conditions, holding a gun can substantially alter the manner in which attention is allocated within scenes.

Why does holding a gun change one's attentional priorities? One set of hypotheses can be postulated that suppose that established mechanisms - including participant expectations, task demands, priming, and unusual or unexpected stimuliunderlie our effects. We find these explanations unlikely for several reasons. First, our manipulations were between subjects. As a result, participants were blind with respect to the full set of manipulations, which limits the influence of 
participant expectations. Second, our task involved freeviewing for all three conditions, which limits the influence of task demands. Only the circumstances under which the observer viewed the stimuli changed, and not anything about the stimuli or the task itself. Third, participants possessed a firearm in both Experiments 2 (held gun) and 3 (holstered gun), but these groups behaved in very distinct ways. In fact, the difference between these experiments suggests that explanations based on priming or unusual stimuli fail. Because participants in both Experiments 2 and 3 were equally aware that they would have a firearm before the experiment began, it is difficult to attribute the observed behavioral differences to either a priming of one's expectations about weapons or the unusualness of a weapon in either particular scenario. Furthermore, we have demonstrated that even when clear expectations can be established for scene content (i.e., the second half of each experiment), the effects of holding a gun remain present, which limits the influence of unusual or unexpected stimuli.

The rejection of these hypotheses leads us to consider a new mechanistic explanation based on an action-attention link. At the outset of this article, we outlined several forms such a link could take. The first was an "all-objects-are-guns hypothesis" based on previous work showing that armed observers are biased to perceive guns in the hands of others (Witt \& Brockmole, 2012). Under this view, we would have expected armed observers to treat scenes with neutral objects in the same way that they treat scenes with guns. The second was a "protection-invincibility" hypothesis, which argues that holding a gun makes you feel safe. In this case, we would have expected observers to treat scenes with guns as if they were more neutral. Both hypotheses, however, can be rejected for the same reason: The weapon focus effect is neither augmented nor reduced when holding a gun. In all cases, the relative difference in attention allotted to weapons and neutral objects was comparable.

A third possible outcome was supported by the data, in which we supposed that holding a gun could affect how individuals view the world in terms of affordances (cf. Gibson, 1979). When holding a gun themselves, observers allocated attention to different sources of information than they did when they were not holding a weapon in a readily usable position. Specifically, armed observers attended less to objects overall and more to faces. When viewed in the context of affordances, this result makes sense. An armed observer must decide if and when to use his or her firearm. In this case, attending to sources of information that more reliably indicate the impending actions of another person is important. Indeed, facial expressions can be strong indicators of behavioral intentions, particularly when a face conveys anger (Horstmann, 2003), and so the observed shift in attention from objects to faces among those holding a firearm likely reflects a shift in information value. It is important to be clear, however, that this shift in information value is derived from the fact that the action capabilities of the observer were altered, since this shift was not observed when the gun was holstered.

This affordance-based explanation also aligns with one proposal derived from a meta-analysis of literature concerning eyewitness testimony (Deffenbacher, Bornstein, Penrod, \& McGorty, 2004). According to this proposal, effects on eyewitness testimony depend upon the response mode elicited by the particular manipulation. Some manipulations induce orienting responses, whereas others induce defensive responses, and defensive responses could enhance or impair memory, depending upon the specific scenario. Our manipulation clearly alters any possible defensive response by providing the observer with a weapon comparable to those wielded by actors in the images. If observers are making an appraisal of whether to use their enhanced defensive capabilities, then the face of the individual is a more potent source of information for that decision than either the background or the weapon in a scene. The result is more time spent looking at faces when the observer holds a weapon than when the observer does not hold a weapon. This pattern of looking behavior could also help explain how a defensive response could enhance memory in this scenario: The more time someone spends looking at the face of a possible assailant, the more likely it is that that observer will remember various details about said assailant.

It is also important to consider how our findings might influence the broader literature on weapon focus. Any weapon is inherently significant in a scene because of the potential danger, but alternative explanations have suggested that the weapon focus effect can also be explained by unusualness (e.g., Pickel, 1998, 1999). These two possibilities are not mutually exclusive, and both could lead to an increase in attention paid to weapons, relative to other objects. Indeed, our experiments showed a significant difference in time spent looking at the perpetrator's face when the perpetrator held a weapon versus a nonweapon, but only during the first half of the experiment. This finding appears to support the idea that unusualness contributes to weapon focus, since the weapon focus effect can be diminished by an observer's expectations. Similar effects have been shown through an eliminated weapon focus effect when observers anticipated seeing a dangerous individual (Pickel, 2009). Although weapon focus is more often studied as a memory effect, the role exposure duration played in our experiments highlights how examining weapon focus through the lens of attention can provide new insights into the effect and its causes.

Beyond the specific context of weapon focus or the appraisal of threat, linking action and attentional control represents a significant addition to our understanding of cognitive control. Other research has suggested that shifts of attention depend upon physical salience (Itti $\&$ Koch, 2000) or depend upon the next action during a 
given task (Hayhoe \& Ballard, 2005). These factors are dependent upon a particular image or the task at hand, respectively, but do not incorporate the specific observer and the corresponding capabilities as a prominent factor in directing attention. Our results suggest that a complete view of attention allocation needs to extend beyond the visual particulars of a scene or the task at hand and should include some consideration of the observer's potential repertoire of actions. In particular, action-specific potential biases an observer to direct attention toward those areas related to the given actionspecific potential. An armed observer will pay more attention to potential threats much as a juggler might pay more attention to a set of juggling balls. Such capabilities exemplify how factors unrelated to stimulus-based differences or a given task can influence attention.

Beyond a theoretical contribution, this research has implications for real-world scenarios involving weapons. The weapon focus literature has greatly contributed to questions concerning the validity of eyewitness testimony, especially when the observer in question is the victim of a crime. In fact, a survey of court judges showed that the majority believed the weapon focus effect reliable enough to be expressed in court as evidence of the unreliability of eyewitness testimony (Wise \& Safer, 2003, 2004). Our findings showed a means by which other aspects of the situation could influence attention allocation and, thus, could also affect memory for events. In particular, Hulse and Memon (2006) used armed police officerswhose weapons could be readily used - and found more accurate, rather than less accurate, recall of a simulated crime, although recall of the event was less complete. Their results may be due not only to the particular population of police officers used in the study, but also to the changed attentional allocation of a police officer with a weapon ready to use. As armed individuals shift their attentional priorities, memory for scene details are also likely to shift. Of course, our concentration on attentional control does not establish a memory effect but provides a strong indication that one might exist. Future research needs to investigate this possibility.

Additionally, it is interesting to consider how well our findings might extend to other weapons or scenarios involving weapons. Simply having a weapon was not enough to generate any difference, since participants with a holstered firearm (Experiment 3) showed no difference from unarmed observers (Experiment 1). If a weapon cannot be used effectively (e.g., the gun is holstered and fastened in place, or an observer with a knife is far away from another individual with a gun), then it seems that attention will be allocated as though the observer was unarmed. Another issue is whether or not the individual intends to use the weapon versus having the weapon in a readily usable position. In previous studies, intention to use a tool facilitated a change in perception even when the tool itself was not being held (Witt \& Proffitt, 2008). In our study, the posture of holding a gun toward the screen potentiated the action even without an explicit intention to immediately use the weapon. This potentiated action may also occur with a holstered gun that an individual is prepared to use. For example, a police officer may leave the gun in his or her holster but may unclip anything fastening the weapon in place and hold a hand ready to remove the firearm. The result could be a change in attentional processing similar to what would occur if the weapon were already drawn. Finally, we should also note that while the provided firearm was nonfunctional, it remained a highly realistic weapon in the highly unusual context of a university laboratory. The juxtaposition of realism and unusualness for our weapon appears to have circumvented the issue of actual functionality, but more important, we were able to show that the key difference was readiness to use the weapon. This combination further contributes to the idea that, like imagination or intent (e.g., Davoli et al., 2012; Witt \& Proffitt, 2008), realism is an important factor in engaging action-specific differences.

In conclusion, the present study shows that the actionspecific potential of the observer can alter attentional allocation. We replicated weapon focus with modern eye-tracking technologies and verified that the increase in looking at weapons does indeed come at a cost of looking less at faces. However, arming the observer with a gun elicited a separate bias to look at faces if the firearm was in a readily usable position. These two biases, weapon focus and the armed bias to look at faces, were similar in magnitude but opposite in direction. The result was not a modification of the weapon focus effect, but a return to baseline performance where armed observers looked at armed actors as much as unarmed observers looked at unarmed actors. The present evidence therefore helps show just how important the capabilities of the observer are in affecting cognitive functioning. Simply holding a gun altered attentional allocation and influenced gaze control decisions. Thus, factors unrelated to a given task or the physical properties of the image are capable of influencing cognitive control.

\section{References}

Antes, J. R. (1974). The time course of picture viewing. Journal of Experimental Psychology, 103, 62-70.

Brockmole, J. R., \& Henderson, J. M. (2006a). Recognition and attention guidance during contextual cueing in real-world scenes: Evidence from eye movements. Quarterly Journal of Experimental Psychology, 59, 1177-1187.

Brockmole, J. R., \& Henderson, J. M. (2006b). Using real-world scenes as contextual cues for search. Visual Cognition, 13, 99-108.

Brockmole, J. R., Davoli, C. C., Abrams, R. A., \& Witt, J. K. (2013). The world within reach: Effects of hand posture and tool use on visual cognition. Current Directions in Psychological Science, 22, 38-44.

Castelhano, M. S., \& Henderson, J. M. (2007). Initial scene representations facilitate eye movement guidance in visual search. Journal of 
Experimental Psychology: Human Perception and Performance, 33, 753-763.

Castelhano, M. S., Mack, M. L., \& Henderson, J. M. (2009). Viewing task influences eye movement control during active scene perception. Journal of Vision, 9(3), 1-15. 6.

Davoli, C. C., Brockmole, J. R., \& Witt, J. K. (2012). Compressing perceived distance with remote tool-use: Real, imagined, and remembered. Journal of Experimental Psychology: Human Perception and Performance, 38, 80-89.

Deffenbacher, K. A., Bornstein, B. H., Penrod, S. D., \& McGorty, E. K. (2004). A meta-analytic review of the effects of high stress on eyewitness memory. Law and Human Behavior, 28, 687-706.

Eckstein, M. P., Drescher, B., \& Shimozaki, S. S. (2006). Attentional cues in real scenes, saccadic targeting and Bayesian priors. Psychological Science, 7, 973-980.

Fawcett, J. M., Russell, E. J., Peace, K. A., \& Christie, J. (2013). Of guns and geese: A meta-analytic review of the 'weapon focus' literature. Psychology, Crime \& Law, 19, 35-66. doi:10.1080/1068316X.2011. 599325

Gibson, J. J. (1979). The ecological approach to visual perception. Boston: Houghton Mifflin.

Gray, R. (2012). Being selective at the plate: Processing dependence between perceptual variables relates to hitting goals and performance. Journal of Experimental Psychology: Human Perception and Performance. doi:10.1037/a0030729

Hayhoe, M. M., \& Ballard, D. H. (2005). Eye movements in natural behavior. Trends in Cognitive Sciences, 9, 188-194.

Hayhoe, M. M., Shrivastava, A., Mruczek, R., \& Pelz, J. B. (2003). Visual memory and motor planning in a natural task. Journal of Vision, 3 , $49-63$.

Henderson, J. M., Weeks, P. A., Jr., \& Hollingworth, A. (1999). Effects of semantic consistency on eye movements during scene viewing. Journal of Experimental Psychology: Human Perception and Performance, 25, 210-228.

Hommel, B., Müsseler, J., Aschersleben, G., \& Prinz, W. (2001). The theory of event coding (TEC): A framework for perception and action planning. Behavioral and brain sciences, 24, 849-878.

Hope, L., \& Wright, D. (2007). Beyond unusual? Examining the role of attention in the weapon focus effect. Applied Cognitive Psychology, 21, 951-961.

Horstmann, G. (2003). What do facial expressions convey: Feeling states, behavioural intentions, or action requests? Emotion, 3, 150-166.

Hulse, L. M., \& Memon, A. (2006). Fatal impact? The effects of emotional arousal and weapon presence on police officers' memories for a simulated crime. Legal and Criminological Psychology, 11, 313-325.

Iriki, A., Tanaka, M., \& Iwamura, Y. (1996). Coding of modified body schema during tool use by macaque postcentral neurones. Neuroreport, 7, 2325.

Itti, L., \& Koch, C. (2000). A saliency-based search mechanism for overt and covert shifts of visual attention. Vision Research, 40, 1489-1506.

Loftus, G. R., \& Mackworth, N. H. (1978). Cognitive determinants of fixation location during picture viewing. Journal of Experimental Psychology: Human Perception and Performance, 4, 565-572.

Loftus, E. F., Loftus, G. R., \& Messo, J. (1987). Some facts about 'weapon focus'. Law and Human Behaviour, 1, 55-62.
Miles, J. D., \& Proctor, R. W. (2011). Colour correspondence effects between controlled objects and targets. The Quarterly Journal of Experimental Psychology, 64, 2044-2064.

Miller, L. (2013, April). Nate Bell, Arkansas state lawmaker, sends insensitive tweet about 'Boston Liberals.' Retrieved from http:// www.huffingtonpost.com/2013/04/19/nate-bell-tweet-boston-_n 3116480.html

Müsseler, J., \& Hommel, B. (1997). Blindness to response-compatible stimuli. Journal of Experimental Psychology: Human Perception and Performance, 23, 861-872. doi:10.1037/0096-1523.23.3.861

Osiurak, F., Morgado, N., \& Palluel-Germain, R. (2012). Tool use and perceived distance: When unreachable becomes spontaneously reachable. Experimental Brain Research, 218(2), 331-339.

Peters, D. P. (1988). Eyewitness memory and arousal in a natural setting. In M. Gruneberg, P. Morris, \& R. Skyes (Eds.), Practical aspects of memory: Current research and issues (Memory in everyday life, Vol. 1, pp. 89-94). Chichester: Wiley.

Pickel, K. L. (1998). Unusualness and threat as possible causes of 'weapon focus'. Memory, 6, 277-295.

Pickel, K. L. (1999). The influence of context on the 'weapon focus' effect. Law and Human Behaviour, 23, 299-311.

Pickel, K. L. (2009). The weapon focus effect on memory for female versus male perpetrators. Memory, 17, 664-678.

Stefanucci, J. K., \& Geuss, M. N. (2009). Big people, little world: The body influences size judgments. Perception, 38, 1782-1795.

Tooley, V., Brigham, J. C., Maass, A., \& Bothwell, R. K. (1987). Facial recognition: Weapon effect and attentional focus. Journal of Applied Social Psychology, 17, 845-859.

Wise, R. A., \& Safer, M. A. (2003). A survey of judges' knowledge and beliefs about eyewitness testimony. Court Review, 40, 6-16.

Wise, R. A., \& Safer, M. A. (2004). What US judges know and believe about eyewitness testimony. Applied Cognitive Psychology, 18, 427-443.

Witt, J. K. (2011). Actions effect on perception. Current Directions in Psychological Science, 20, 201-206.

Witt, J. K., \& Brockmole, J. R. (2012). Action alters object identification: Wielding a gun increases the bias to see guns. Journal of Experimental Psychology: Human Perception and Performance, 38, 1159-1167.

Witt, J. K., \& Proffitt, D. R. (2005). See the ball, hit the ball apparent ball size is correlated with batting average. Psychological Science, 16, 937-938.

Witt, J. K., \& Proffitt, D. R. (2008). Action-specific influences on distance perception: A role for motor simulation. Journal of Experimental Psychology: Human Perception and Performance, 34, 1479-1492.

Witt, J. K., Proffitt, D. R., \& Epstein, W. (2005). Tool use affects perceived distance, but only when you intend to use it. Journal of Experimental Psychology: Human Perception and Performance, 31, 880-888.

Witt, J. K., Kemmerer, D., Linkenauger, S. A., \& Culham, J. (2010). A functional role for motor simulation in identifying tools. Psychological Science, 21, 1215-1219.

Yarbus, A. L. (1967). Eye movements and vision. New York: Plenum Press.

Zwickel, J., Grosjean, M., \& Prinz, W. (2007). Seeing while moving: Measuring the online influence of action on perception. Quarterly Journal of Experimental Psychology, 60, 1063-1071. doi:10.1080/ 17470210701288722 